\title{
AUTOMORPHISMS OF FUNCTION FIELDS
}

\author{
BY \\ MAXWELL ROSENLICHT
}

1. Let $K$ be an algebraic function field of one variable over the constant field $k$ and let $g>0$ be the genus of $K$. Let $G$ be the group of all automorphisms of $K$ that leave the elements of $k$ fixed (and that leave a given place $P_{0}$ of $K / k$ fixed if $g=1$ ). A classical theorem due to Schwartz-Klein-NoetherWeierstrass-Poincaré-Hurwitz when $g>1$ (and older for $g=1$ ) says that $G$ is finite if $k$ is the field of complex numbers. From this one can easily deduce the same result if $k$ is any field of characteristic zero. The theorem for $k$ an algebraically closed field of characteristic $p \neq 0$ was proved by $H$. L. Schmid in 1938 [5], and a less computational proof for any algebraically closed $k$ was given recently by Iwasawa and Tamagawa [3]. We intend to show how this result can be very easily proved by one of the classical arguments (given in essence, but somewhat imprecisely, in [1]) if we replace integration on the Riemann surface $R$ of $K$ by use of its jacobian variety $J$, and finally we shall show what the corresponding result is when $k$ is an arbitrary field. The reasons for including here the easy case $g=1$ will become apparent in the last section.

The analytic proof we have in mind runs as follows: $G$ is naturally isomorphic to the group of complex analytic homeomorphisms of $R$ (that leave $P_{0}$ fixed if $g=1$ ). First consider the special case in which $R$ is elliptic or hyperelliptic. $R$ can then be considered (in one and only one way) as a two-sheeted covering surface of a Riemann sphere $S$ (such that, if $g=1, P_{0}$ is a branch point of this covering). The elements of $G$ give rise to analytic homeomorphisms of $S$ that permute the ramification points of $S$. Since $g>0$, the ramification points are in finite number $>2$. The finiteness of $G$ then follows from (1) any analytic homeomorphism of $S$ leaving three distinct points fixed is the identity, and (2) any element of $G$ that leaves all points of $S$ fixed is either the identity or merely interchanges the sheets of $R$. On the other hand if $K$ is not elliptic or hyperelliptic, then the ratios of the differentials of the first kind of $K$ give rise to the canonical embedding of $R$ in $S_{o-1}$, the complex projective space of dimension $(g-1)$, and the automorphisms of $K / k$ correspond one-one to projective transformations of $S_{g-1}$ that map $R$ onto itself. It follows that $G$ can be considered as a Lie group with a finite number of components that acts analytically on $R$ (see the second lemma of $\$ 2$ for details), so it remains only to show that the component of the identity $G$ of $G$ has only one point. Hence we have to show that if $\sigma_{1}, \sigma_{2} \in G$ are homotopic (as maps of $R$ ), then $\sigma_{1}=\sigma_{2}$. So let $\omega$ be any differential of the first kind on $R$

Presented to the Society, December 28, 1953; received by the editors January 16, 1954. 
and let $\Gamma$ be any 1-cycle on $R$. Then $\sigma_{1}^{-1}(\Gamma)$ is homologous to $\sigma_{2}^{-1}(\Gamma)$, and hence $\int_{\Gamma} \sigma_{1}(\omega)=\int_{\sigma_{1}}{ }^{-1}(\Gamma) \omega=\int_{\sigma_{2}}{ }^{-1}(\Gamma) \omega=\int_{\Gamma} \sigma_{2}(\omega)$. Thus all the periods of $\sigma_{1}(\omega)$ $-\sigma_{2}(\omega)$ are zero, so $\sigma_{1}(\omega)=\sigma_{2}(\omega)$. Since this is true for each $\omega$ and since the quotients of the $\omega$ 's generate $K$, we have $\sigma_{1}=\sigma_{2}$. Q.E.D.

2 . In this section $k$ is supposed algebraically closed.

LEMMA. If $K / k$ is any algebraic function field of one variable, there exists a nonsingular algebraic curve $C$ in a projective space $S_{n}$ such that $C$ is defined over $k$, its function field $k(C)$ is $k$-isomorphic to $K$, and each birational map of $C$ onto itself (that leaves a given place $P_{0}$ of $K$ fixed if $g=1$ ) is induced by a nonsingular projective transformation of $S_{n}$.

We prove this lemma generally to avoid the necessity for special consideration of the hyperelliptic case, which is messy in the case of characteristic 2. We first assume $g>1$ and show that the tricanonical image of $K$ will do the trick. Let $W_{1}, W_{2}, W_{3}$ be canonical divisors of $K$. Then $d\left(W_{1} W_{2} W_{3}\right)=6 g-6$ $>2 g-2$, so $i\left(W_{1} W_{2} W_{3}\right)=0$ and the Riemann-Roch theorem gives $r\left(W_{1} W_{2} W_{3}\right)$ $=5 g-5$. Let $f_{1}, \cdots, f_{5 g-5} \in K$ be a basis for the vector space $L\left(W_{1} W_{2} W_{3}\right)$ of multiples of $\left(W_{1} W_{2} W_{8}\right)^{-1}$. Since any two canonical divisors of $K$ are linearly equivalent, if we started with different canonical divisors $W_{1}^{\prime}, W_{2}^{\prime}, W_{3}^{\prime}$ we could replace $f_{1}, \cdots, f_{b_{0}-6}$ by their multiples by a certain nonzero element of $K$. It follows that the algebraic curve $C$ defined over $k$ by the homogeneous generic point $\left(f_{1}, \cdots, f_{50-5}\right)$, which is embedded in the projective space $S_{60-6}$ of dimension $5 g-6$, is invariantly defined by $K$ to within nonsingular projective transformations with coefficients in $k$ of $S_{50-6} . k(C)$ $=k\left(\left\{f_{i} / f_{j}\right\}\right), i, j=1, \cdots, 5 g-5$, so $k(C) \subseteq K$. We now show that $k(C)=K$. $K$ has precisely $g$ linearly independent differentials of the first kind, so we can find distinct places $P_{1}, \cdots, P_{o}$ of $K$ such that $i\left(P_{1} \cdots P_{0}\right)=0$. For each $j=1, \cdots, g$, we have $i\left(P_{1} \cdots P_{o} P_{j}^{-1}\right)=1$. Choose distinct places $P^{\prime}, P^{\prime \prime}$ that are distinct from the zeros of the differentials that are multiples of the various divisors $P_{1} \cdots P_{0} P_{j}^{-1}$. Then each integral divisor of degree $g$ that divides $P_{1} \cdots P_{0} P^{\prime}$ is nonspecial, and similarly for $P_{1} \cdots P_{0} P^{\prime \prime}$. Hence, by Riemann-Roch, there exist functions $g_{1}, g_{2} \in K$ whose polar divisors are $P_{1} \cdots P_{0} P^{\prime}$ and $P_{1} \cdots P_{0} P^{\prime \prime}$ respectively. Now choose nonzero differentials of first kind $\omega_{1}, \omega_{2}, \omega_{3}$ such that $P_{1} \cdots P_{g-1}\left|\left(\omega_{1}\right), P_{g}\right|\left(\omega_{2}\right), P^{\prime} \mid\left(\omega_{3}\right)$. Setting $W_{i}=\left(\omega_{i}\right), i=1,2,3$, we get $P_{1} \cdots P_{0} P^{\prime} \mid W_{1} W_{2} W_{3}$. Hence $1, g_{1}$ $\in L\left(W_{1} W_{2} W_{3}\right)$, so $g_{1} \in k(C)$. Similarly $g_{2} \in k(C)$. For suitable $c \in k, g_{1}+c g_{2}$ has polar divisor $P_{1} \cdots P_{\theta} P^{\prime} P^{\prime \prime}$; since $\left[K: k\left(g_{1}\right)\right]=g+1,\left[K: k\left(g_{1}+g_{2}\right)\right]=g+2$, we get $k(C)=K$. Next let $P_{1}, P_{2}$ be any places of $K$, not necessarily distinct, and choose integral canonical divisors $W_{1}, W_{2}, W_{3}$ prime to $P_{1}$. Then $d\left(W_{1} W_{2} W_{3} P_{1}^{-1} P_{2}^{-1}\right)=6 g-8>2 g-2$, so $i\left(W_{1} W_{2} W_{3} P_{1}^{-1} P_{2}^{-1}\right)=0$. Thus $r\left(W_{1} W_{2} W_{3} P_{1}^{-1}\right)=r\left(W_{1} W_{2} W_{3} P_{1}^{-1} P_{2}^{-1}\right)+1$, and there exists $f \in L\left(W_{1} W_{2} W_{8}\right)$ such that $P_{1} \mid(f)_{0}, P_{1} P_{2} \nmid(f)_{0}$. Since each function in our present $L\left(W_{1} W_{2} W_{3}\right)$ is finite at $P_{1}$, this implies the nonsingularity of $C$. Any birational map of $C$ 
onto itself that is defined over $k$ comes from a $k$-automorphism of $K$, which can merely permute the canonical divisors of $K$, so this birational map comes from a nonsingular projective transformation of $S_{5 q-6}$; if we have a birational map of $C$ onto itself that is not defined over $k$, we merely extend the constant field $k$ to get the same result, and this finishes the case $g>1$. If $g=1$, we have $L\left(P_{0}^{\nu}\right)=\nu$ for $\nu>0$, so there exist $x, y \in K$ such that $(1, x)$ and $(1, x, y)$ are bases for $L\left(P_{0}^{2}\right)$ and $L\left(P_{0}^{3}\right)$ respectively. $[K: k(x)]=2,[K: k(y)]=3$, so $K=k(x, y)$. If $C$ is the curve in $S_{2}$ having as homogeneous generic point over $k$ the point $(1, x, y)$, then $k(C)=K$. The seven quantities $y^{2}, y x, y, x^{3}, x^{2}, x$, $1 \in L\left(P_{0}^{6}\right)$ (a space of dimension 6), so $C$ is a cubic curve. $C$ is nonsingular, for otherwise it would be rational. For any birational map $\sigma$ of $C$ onto itself such that $\sigma\left(P_{0}\right)=P_{0}$ each space $L\left(P_{0}^{\nu}\right)$ is invariant under $\sigma$, so $\sigma(x)=a+b x$, $\sigma(y)=c+d x+e y$, where $a, \cdots, e$ are constants and $b e \neq 0$. This ends the case $g=1$. If $g=0$, take $C=S_{1}$. Q.E.D.

Lemma. If $C$ is a nonsingular curve of genus $g$, there exists an algebraic group variety $G$ which may be identified with a subgroup of finite index of the group of all birational transformations of $C$ onto itself (that leave a given point $P_{0} \in C$ fixed if $\left.g=1\right)$ such that the map $\Psi: G \times C \rightarrow C$ defined by $\Psi(\sigma \times P)=\sigma(P)$ is an everywhere defined rational map.

Let $k$ be an algebraically closed field of definition for $C$ and let $C$ be the curve of the preceding lemma. Let $Y_{0}, \cdots, Y_{n}$ be projective coordinates of $S_{n}$. Then any birational map $\sigma$ of $C$ onto itself (which leaves $P_{0}$ fixed if $g=1)$ is induced by a projective transformation $Y_{i} \rightarrow \sum_{j=0}^{n} c_{i j} Y_{j}$, where $\left(c_{i j}\right)$ is a nonsingular matrix of order $(n+1)$ with constant coefficients. (So $\left|c_{i j}\right| \neq 0$.) Choose the integer $N$ so large that the forms in $k[Y]$ of degree $N$ which vanish on $C$ actually define $C$, and let $F_{1}, \cdots, F_{m}, F_{m+1}, \cdots, F_{M}$ $\in k[Y]$ be a basis for all forms of degree $N$ such that the subspace spanned by $F_{1}, \cdots, F_{m}$ consists precisely of all forms of degree $N$ vanishing on $C$. The matrix $\left(c_{i j}\right)$ then gives rise to a linear transformation of the vector space with basis elements $F_{1}, \cdots, F_{M}$,

$$
\left(c_{i j}\right): F_{\beta} \rightarrow \sum_{\alpha=1}^{M} A_{\beta \alpha}\left(\left(c_{i j}\right)\right) F_{\alpha} \quad(\beta=1, \cdots, M),
$$

where the $A_{\beta \alpha}$ 's are forms in $k\left[\left\{c_{i j}\right\}\right]$. The conditions that $\left(c_{i j}\right)$ map $C$ into itself are then $A_{\beta \alpha}\left(\left(c_{i j}\right)\right)=0, \beta=1, \cdots, m, \alpha=m+1, \cdots, M$. Conversely, if $\left|c_{i j}\right| \neq 0$ and $\left(c_{i j}\right)$ satisfies these last conditions it induces a birational map of $C$ onto itself. (If $g=1$, we must add the further algebraic condition $\left(c_{i j}\right): P_{0} \rightarrow P_{0}$.) We may clearly assume that $C$ spans $S_{n}$. Then two $\left(c_{i j}\right)$ 's give rise to the same birational transformation of $C$ if and only if they are proportional. Thus the birational transformations of $C$ (which leave $P_{0}$ fixed if $g=1$ ) may be identified with the points of an abstract algebraic variety $G^{\prime}$ (here an algebraic variety minus a subvariety) in $S_{(n+1)}{ }^{2}-1 . G^{\prime}$ is a group 
under matrix multiplication, which corresponds to the composition of birational maps. We have only to take $G$ to be the component of the identity of $G^{\prime}$. Q.E.D.

THEOREM. Let $K$ be an algebraic function field of one variable over the algebraically closed constant field $k$. If $K$ has genus $g>0$, then the group $G$ of all $k$-automorphisms of $K$ (which leave a given place $P_{0}$ of $K$ fixed if $g=1$ ) is finite.

Let $C$ be a nonsingular projective model of $K / k$. Then it suffices to show that the group of birational transformations of $C$ onto itself (or the subgroup of these leaving $P_{0}$ fixed if $g=1$ ) is finite. It suffices to show that if $C, G$ are as in the preceding lemma, then $G=e$ (=the identity map). If $g>1$, fix some point $P_{0} \in C$. Let $\phi$ be the canonical map of $C$ into its jacobian variety $J$, normalized so that $\phi\left(P_{0}\right)=0$ (cf. [7]). Since $J$ is an abelian variety we can write $\phi \Psi(\sigma \times P)=\psi(\sigma)+\psi^{\prime}(P)$, where $\psi, \psi^{\prime}$ are rational maps of $G$ and $C$ respectively into $J$, and where we may suppose that $\psi(e)=0$. Thus $\phi \sigma(P)$ $=\psi(\sigma)+\psi^{\prime}(P)$. Setting $\sigma=e$, we get $\psi^{\prime}(P)=\phi(P)$. Setting $P=P_{0}$ gives $\psi(\sigma)$ $=\phi \sigma\left(P_{0}\right)$. Hence

$$
\phi \sigma(P)=\phi \sigma\left(P_{0}\right)+\phi(P) .
$$

If $\sigma\left(P_{0}\right)$ is not constant we get $\phi(C)+\phi(C) \subseteq \phi(C)$. Since $\phi(C)$ generates $J$, we must have $\phi(C)=J$. Since $\phi(C)$ is a curve and $J$ has dimension $g$, we have a contradiction in the case $g>1$ unless $\sigma\left(P_{0}\right)=e\left(P_{0}\right)=P_{0}$; if $g=1$, we have $\sigma\left(P_{0}\right)=P_{0}$ by assumption. Thus $\phi \sigma(P)=\phi\left(P_{0}\right)+\phi(P)=\phi(P)$. Hence the divisor $\sigma(P) P^{-1}$ is principal. Since $g>0$, we must have $\sigma(P)=P$, so $\sigma=e$. Q.E.D.

[REMARK. The above argument can be modified slightly to give the following known result, which is the essence of our proof: An irreducible algebraic system of rational endomorphisms of an abelian variety consists of only one endomorphism.]

3. In this section we let $K$ be a field of algebraic functions of one variable of genus $g>0$ over the arbitrary constant field $k$. Let $G$ be the group of $k$ automorphisms of $K$ if $g>1$; if $g=1$, let $G$ be the group of $k$-automorphisms of $K$ leaving fixed a given place $P_{0}$ of $K$. If $G$ is infinite we say that $K$ satisfies the exceptional case. We proceed to give a full account of the exceptional case.

Lemma. Let $E$ be any field, $G$ a group of automorphisms of $E$, and let $F$ be the subfield of $E$ consisting of all elements of $E$ left fixed by each automorphism of $G$. Then $E$ is separably generated over $F$.

This has content only if $E$ has characteristic $p \neq 0$. We have to show that if we have a relation $\sum_{i=1}^{n} c_{i} f_{i}^{p}=0$, where each $c_{i} \in F$ and each $f_{i} \in E$ and where not all the $c_{i}$ 's are 0 , then $f_{1}, \cdots, f_{n}$ are linearly dependent over $F$. Clearly we may take $n>1$. If $\sigma_{1}, \cdots, \sigma_{n} \in G$, we have $\sum_{i=1}^{n} c_{i} \sigma_{j}\left(f_{i}^{p}\right)=0$, $j=1, \cdots, n$, so $\left|\sigma_{j}\left(f_{i}^{p}\right)\right|_{i, j=1, \cdots, n}=0$, and hence $\left|\sigma_{j}\left(f_{i}\right)\right|_{i, j=1, \cdots, n}=0$. Let $r$ 
be the maximal rank that $\left(\sigma_{j}\left(f_{i}\right)\right)_{i, j=1, \ldots, n}$ can assume for $\sigma_{1}, \cdots, \sigma_{n} \in G$; then $1 \leqq r<n$. Reorder the $f_{i}$ 's and choose $\sigma_{1}, \cdots, \sigma_{r} \in G$ so that $\left|\sigma_{j}\left(f_{i}\right)\right|_{i, j=1, \ldots, r} \neq 0$. Hold $\sigma_{1}, \cdots, \sigma_{r}$ fixed and let $\sigma_{r+1} \in G$ be arbitrary. Then $\left|\sigma_{j}\left(f_{i}\right)\right|_{i, j=1, \cdots, r+1}=0$, so there exist $h_{1}, \cdots, h_{r} \in E$ such that $\sigma_{j}\left(f_{r+1}\right)$ $=\sum_{i=1}^{r} h_{i} \sigma_{j}\left(f_{i}\right), j=1, \cdots, r+1$, and $h_{1}, \cdots, h_{r}$ are unique (i.e. independent of the choice of $\left.\sigma_{r+1}\right)$. Thus for any $\sigma \in G$ we have $\sigma\left(f_{r+1}\right)$ $=\sum_{i=1}^{r} h_{i} \sigma\left(f_{i}\right)$. If $\bar{\sigma} \in G$, we have $\sigma\left(f_{r+1}\right)=\bar{\sigma} \bar{\sigma}^{-1} \sigma\left(f_{r+1}\right)=\bar{\sigma} \sum_{i=1}^{r} h_{i} \bar{\sigma}^{-1} \sigma\left(f_{i}\right)$ $=\sum_{i=1}^{r} \bar{\sigma}\left(h_{i}\right) \sigma\left(f_{i}\right)$. By the unicity of $h_{1}, \cdots, h_{r}$, we have $\bar{\sigma}\left(h_{i}\right)=h_{i}$, so each $h_{i} \in F$. Hence $f_{1}, \cdots, f_{n}$ are linearly dependent over $F$.

COROLLARY. If $K$ is an arbitrary algebraic function field of one variable with constant field $k$ ( $K$ possibly of genus zero) and if $K$ possesses an infinite number of $k$-automorphisms, then $K$ is separably generated over $k$.

For the subfield of $K$ left element-wise fixed by each $k$-automorphism of $K$ must contain $k$ and be of infinite index under $K$. Hence this subfield is $k$ itself.

Now let $K / k$ be such that the exceptional case holds. Then $K$ is separably generated over $k$. If $k^{\prime}$ is any algebraic extension of $k$ we can define $k^{\prime} K$, which is a function field of one variable with constant field $k^{\prime}$. Any place of $k^{\prime} K$ lies over a unique place of $K$ and over any place of $K$ lies exactly one place of $k^{\prime} K$. (By a place of $K / k$ we mean a $k$-homomorphism of a valuation ring of $K$ into a fixed algebraic closure of $k$.) Any automorphism $\sigma \in G$ induces a $k^{\prime}$-automorphism of $k^{\prime} K$, so $k^{\prime} K$ has an infinity of $k^{\prime}$-automorphisms. Let the curve $C$ be a projective model of $K / k$ each point of which is simple with reference to $k$. Then $C$ has only a finite number of points that are not absolutely simple, and these correspond to a finite number of distinct places $P_{1}, \cdots, P_{s}$ of $K$. Such places we call singular places of $K$; the residue class field of $K$ at each place $P_{i}$, denoted by $k\left(P_{i}\right)$, must be inseparable over $k$ (cf. [8]). Clearly the places $P_{1}, \cdots, P_{s}$ must be permuted among themselves by each $\sigma \in G$. Thus each $k^{\prime}$-automorphism of $k^{\prime} K$ corresponding to any $\sigma \in G$ must permute the places of $k^{\prime} K$ lying over $P_{1}, \cdots, P_{s}$. The genus of $k^{\prime} K$ is $\leqq g$, with equality if $s=0$ or if $k^{\prime}$ is separable over $k[4 ; 2]$, so that $k^{\prime} K / k^{\prime}$ either satisfies the exceptional case or has genus zero. But the exceptional case cannot arise if the constant field is algebraically closed, so $\bar{k} K / \bar{k}$ must be rational. ( $\bar{k}$ denotes the algebraic closure of $k$.) Hence $s>0$ and $K$ has characteristic $p \neq 0$.

Lemma. Let $K / k$ satisfy the exceptional case. Then there exists a place $P$ of $K$ and a subgroup $\Gamma$ of the group of all $k$-automorphisms of $K$ such that

(1) $\Gamma$ is of finite index in the group of all k-automorphisms of $K$.

(2) Each $\sigma \in \Gamma$ leaves $P$ fixed, and if $\sigma \in \Gamma, \sigma \neq e$, then $P$ is the only place of $K$ left fixed by $\sigma$.

Proof. Let $k^{\prime}$ be any algebraic extension field of $k$ such that $k^{\prime} K / k^{\prime}$ also satisfies the exceptional case. Suppose that $P^{\prime}$ is a place of $k^{\prime} K$ and $\Gamma^{\prime}$ a sub- 
group of the group of all $k^{\prime}$-automorphisms of $k^{\prime} K$, such that (1) and (2) hold for $k^{\prime} K, P^{\prime}, \Gamma^{\prime}$. Then we have our theorem for $K / k$ proved if we let $P$ be the place of $K$ lying below $P^{\prime}$ and let $\Gamma$ consist of all $\sigma \in \Gamma^{\prime}$ that come from $k$-automorphisms of $K$. Hence it suffices to prove our theorem for $k^{\prime} K / k^{\prime}$, provided $k^{\prime} K / k^{\prime}$ has genus $>0$. The genus of $K$ drops to zero when we extend $k$ to $\bar{k}$, hence when we extend $k$ to $k^{p^{-\infty}}$, hence when we extend $k$ to $k^{p^{-\nu}}$, for some integer $\nu$. If we choose $\nu$ minimal and set $k^{\prime}=k^{p^{-(\nu-1)}}$, then $k^{\prime} K / k^{\prime}$ has genus $>0$, while $\left(k^{\prime}\right)^{1 / p} K /\left(k^{\prime}\right)^{1 / p}$ has genus zero. Hence we may assume that $k^{1 / p} K / k^{1 / p}$ has genus zero. If we now let $k^{\prime}$ be the part of $\bar{k}$ that is separably over $k$, then $k^{\prime} K$ has the same genus as $K$ while $\left(k^{\prime}\right)^{1 / p} K$ is still of genus zero. Hence we may assume to begin with that $k^{1 / p} K$ has genus zero and that $k$ is separable algebraically closed. Then $k^{1 / p} K / k^{1 / p} \cong k K^{p} / k$, so the subfield $k K^{p}$ of $K$ has genus zero. Since $k$ is separably algebraically closed, $K$ has a place of degree one, hence so has $k K^{p}$, so $k K^{p}$ is rational. Write $k K^{p}=k(y)$, for some $y \in K$. If $x$ is any separating variable for $K / k$ then each element of $K$ is both separable and purely inseparable over $k(x, y)$, so $K=k(x, y) . x \notin k(y), x^{p} \in k(y)$, so $[K: k(y)]=p$. Any $k$-automorphism $\sigma$ of $K$ induces a $k$-automorphism of $k K^{p}=k(y)$, so $\sigma(y)=(a y+b) /(c y+d)$, where $a, b, c, d \in k$ and $a d \neq b c$. Furthermore, since $K^{p} \subseteq k(y)$, the action of $\sigma$ on $y$ completely determines $\sigma$. Let $P$ be a fixed singular place of $K$. Then the group $H$ of all $k$-automorphisms $\sigma$ of $K$ such that $\sigma(P)=P$ is of finite index in the group of all $k$-automorphisms of $K$, so we may restrict our $\sigma$ 's to $H$. First suppose that $P(y)=\alpha \notin(k, \infty)$. Then $\alpha$ is inseparable over $k$. For each $\sigma \in H$ we have $c \alpha^{2}+d \alpha=a \alpha+b$, so we must have $p=2, d=a, b=c \alpha^{2}, \alpha^{2} \in k$. Hence $\sigma(y)=\left(a y+c \alpha^{2}\right) /(c y+a)$. If $\sigma \in H, \sigma \neq e$, we have $c \neq 0$, so $P$ is the only place of $K$ left fixed by $\sigma$. Thus if we set $\Gamma=H$ we are done in our special case. Hence we may suppose that $P(y) \in(k, \infty)$, and hence that $P(y)=0$. Then for $\sigma \in H$ we have $\sigma(y)=a y /(1+c y), a, c \in k, a \neq 0 . P$ is the only place of $K$ lying over the place $(y=0)$ of $k(y)$, so if $e, f$ are the ramification index and residue class field degree respectively of $P$ over $k(y)$, then $e f=p$. If $f=1$, then $P$ is a place of degree one of $K$, hence nonsingular, contrary to assumption. Thus $f=p, e=1$, so $v_{P}(y)=1$. We now choose $x \in K$ such that $x \notin k(y)$ and $x^{p}=f(y) \in k[y]$, where we suppose that the degree $n$ of the polynomial $f(y)$ is minimal for all such $x . n>0$. If $\sigma \in H$, then

$$
(\sigma(x))^{p}=f(\sigma(y))=f\left(\frac{a y}{1+c y}\right) .
$$

Choose the integer $i$ such that $(i-1) p<n \leqq i p$. Then $i>0$ and

$$
\left((1+c y)^{i} \sigma(x)\right)^{p}=(1+c y)^{p i} f\left(\frac{a y}{1+c y}\right) \in k[y] .
$$

Now $P\left((1+c y)^{i} \sigma(x)\right)=P(\sigma(x))=P(x)$, since $\sigma \in H$, so $v_{P}\left((1+c y)^{i} \sigma(x)-x\right)>0$. 
Hence the only pole of $\left((1+c y)^{i} \sigma(x)-x\right) / y$ is at $(y=\infty)$, and thus

$$
\left(\frac{(1+c y)^{i} \sigma(x)-x}{y}\right)^{p}=\frac{(1+c y)^{p i} f(a y /(1+c y))-f(y)}{y^{p}}
$$

$=$ a polynomial in $y$ of degree $\leqq p i-p<n$. By the minimality property of $n$, $\left((1+c y)^{i} \sigma(x)-x\right) / y \in k[y]$, so we can write $(1+c y)^{i} \sigma(x)=x+h(y)$, with $h(y)$ $\in k[y]$, and we deduce

$$
f(y)+(h(y))^{p}=(1+c y)^{i p} f\left(\frac{a y}{1+c y}\right) .
$$

Differentiating,

$$
f^{\prime}(y)=a(1+c y)^{i p-2} f^{\prime}\left(\frac{a y}{1+c y}\right) .
$$

Since $K$ is separably generated over $k, f^{\prime}(y) \neq 0$, so we write $f^{\prime}(y)=y^{r} u(y)$, where $r \geqq 0$ and $u(y) \in k[y], u(0) \neq 0$. Thus

$$
u(y)=a^{r+1}(1+c y)^{i p-2-r} u\left(\frac{a y}{1+c y}\right) .
$$

Setting $y=0$ gives $a^{r+1}=1$, so there are only a finite number of possibilities for $a$. If we let $\Gamma$ consist of all $\sigma \in H$ with $a=1$, properties (1), (2) follow immediately. Q.E.D.

LEMMA. If $K / k$ satisfies the exceptional case, then $K$ has precisely one singular place $P$ and the residue class field $k(P)$ is purely inseparable over $k . G$ contains a normal subgroup $G$ such that

(1) $G / G$ is cyclic and of finite order prime to $p$.

(2) If $\sigma \in G, \sigma \neq e$, then $\sigma(P)=P$ and $P$ is the only place of $K$ left fixed by $\sigma$.

(3) $G$ is commutative and each of its elements has order $p$. If $g=1$, then $P_{0}=P$ and $G$ is the group of all $k$-automorphisms of $K$.

Proof. We already know that $K$ has at least one singular place $P$, and at most a finite number, and that each $k$-automorphism of $K$ permutes the singular places, so the preceding lemma implies the first statement. If $k(P)$ were not purely inseparable over $k$ we could let $k^{\prime}$ be the separable part of $k(P)$ and then the field $k^{\prime} K / k^{\prime}$ would satisfy the exceptional case and have more than one singular place. The final statement also follows from the previous lemma. Since $P$ is the only singular place of $K$, for each $\sigma \in G$ we have $\sigma(P)=P$. We can write $\bar{k} K=\bar{k}(z)$, where $z$ is infinite at $P$. Then $\sigma(z)=a z+b$, where $a, b \in \bar{k}, a \neq 0$, and $a, b$ completely determine $\sigma$. Let $G$ be the kernel of the homomorphism $\sigma \rightarrow a$ of $G$ into the multiplicative group of $\bar{k}$. Then $G$ consists precisely of $e$ and of all $\sigma \in G$ such that $P$ is the only place left fixed 
by $\sigma$, verifying (2). $G$ contains the $\Gamma$ of the previous lemma, which implies (1). If $\sigma \in G$, then $\sigma(z)=z+b$, which gives (3). Q.E.D.

Now let $K / k$ be any function field satisfying the exceptional case, and let $P, G$ be as in the last lemma. Let $G_{0}$ be a finite subgroup of $G$ of order $p^{n}$ and let $K_{0} \supset k$ be the field of elements of $K$ left fixed by each automorphism of $G_{0}$. If $t \in K_{0}, \sigma_{0} \in G_{0}, \sigma \in G$, then $\sigma_{0}(\sigma(t))=\sigma\left(\sigma_{0}(t)\right)=\sigma(t)$, so $\sigma(t) \in K_{0}$. Thus each $\sigma \in G$ induces an automorphism of $K_{0}$. Furthermore $K$ is a normal separable extension of $K_{0}$ of degree $p^{n}$. Consider Zeuthen's formula, $2 g-2=p^{n}\left(2 g_{0}-2\right)$ $+d(D)$, where $g_{0}$ is the genus of $K_{0}$ and $D$ the different of $K$ with respect to $K_{0}$. Let $P_{0}$ be the place of $K_{0}$ lying under $P$. If the place $P^{\prime}$ of $K$ lies over $P_{0}$ and $P^{\prime} \neq P$, then for any $\sigma_{0} \in G_{0}$ the place $\sigma_{0}\left(P^{\prime}\right)$ lies over $P_{0}$ and the various places $\sigma_{0}\left(P^{\prime}\right)$ (for $\sigma_{0}$ ranging over $G_{0}$ ) are distinct from each other and from $P$; this implies that at least $\left(p^{n}+1\right)$ distinct places of $K$ lie over $P_{0}$, contradicting $\left[K: K_{0}\right]=p^{n}$. Hence $P$ is the only place of $K$ lying over $P_{0}$. Next let $P^{\prime}$ be any place of $K$ distinct from $P$. Then the various places $\sigma_{0}\left(P^{\prime}\right)$ (for $\sigma_{0}$ ranging over $G_{0}$ ) are distinct and in number $p^{n}$ and all lie over the same place of $K_{0}$; it follows that each ramification index and each residue class field degree of each $\sigma_{0}\left(P^{\prime}\right)$ over $K_{0}$ is 1 , so $P^{\prime} \nmid D$. Hence we can write $D=P^{r}$, for some $r \geqq 0$. If $e$ and $f$ are the ramification index and residue class field degree respectively for $P$ over $P_{0}$, then $e f=p^{n}$. If $n>0$ then either $p \mid f$ (so $k(P)$ is inseparable over $k\left(P_{0}\right)$ ) or $p \mid e$, and hence (by [2, p. 69]) $P^{e} \mid D$. Hence $D=P^{r}$, with $r \geqq e$. Thus $2 g-2-p^{n}\left(2 g_{0}-2\right)=r d(P) \geqq e f d\left(P_{0}\right)=p^{n} d\left(P_{0}\right)$. Hence $2 g-2 \geqq p^{n}\left(2 g_{0}-2+d\left(P_{0}\right)\right)$. Thus if $n$ is sufficiently large we have $g_{0}=0$ and $d\left(P_{0}\right) \leqq 2$. Take $n$ so large that this is true. Then if $d\left(P_{0}\right)=2$ we must have $p=2$, and we can find a subgroup $G_{0}^{\prime}$ of $G$ such that $G_{0}^{\prime} \supset G_{0}$ and $G_{0}^{\prime} / G_{0}$ has order 2. Let $K_{0}^{\prime}$ be the subfield of $K$ consisting of all elements left fixed by each $\sigma_{0}^{\prime} \in G_{0}^{\prime}$. Then $K_{0}$ is separable over $K_{0}^{\prime}$ and $\left[K_{0}: K_{0}^{\prime}\right]=2$. Let $P_{0}^{\prime}$ be the place of $K_{0}^{\prime}$ lying under $P_{0} . P_{0}$ is the only place of $K_{0}$ lying over $P_{0}^{\prime}$. Let $e^{\prime}, f^{\prime}$ be the ramification index and residue class field degree respectively for $P_{0}$ over $P_{0}^{\prime}$, and let $D^{\prime}$ be the different of $K_{0} / K_{0}^{\prime}$. By Zeuthen's formula, $d\left(D^{\prime}\right)=2$. If $e^{\prime}=2$ then $P_{0}^{2} \mid D^{\prime}$, so $d\left(D^{\prime}\right) \geqq 4$, which is false, so $e^{\prime} \neq 2$. But $e^{\prime} f^{\prime}=2$, so we have $e^{\prime}=1, f^{\prime}=2$, so $d\left(P_{0}^{\prime}\right)=1$. As a result, if $n$ is sufficiently large we certainly have $g_{0}=0$ and $d\left(P_{0}\right)=1$. Here we can write $K_{0}=k(x)$, where $v_{P_{0}}(x)=-1$.

Fix a subgroup $G_{0}$ of $G$ of least possible order $p^{n}$ such that the fixed field $K_{0}$ of $G_{0}$ is of the form $K_{0}=k(x)$, where $v_{P_{0}}(x)=-1, P_{0}$ being the place of $K_{0}$ under $P$, and let $\sigma_{1}, \cdots, \sigma_{n}$ be a set of generators for $G_{0}$. For $i=1, \cdots, n$ let $G_{i}$ be the subgroup of $G_{0}$ generated by $\sigma_{1}, \cdots, \sigma_{i-1}, \sigma_{i+1}, \cdots, \sigma_{n}$, and let $K_{i}$ be the fixed field of $G_{i}$. $K_{i}$ is a normal extension of $k(x)$ of degree $p$ and the restriction of $\sigma_{i}$ to $K_{i}$ generates the Galois group of $K_{i}$ over $k(x)$. Hence we can find a $y_{i} \in K_{i}$ such that $\sigma_{i}\left(y_{i}\right)=y_{i}+1$, and we have $y_{i}^{p}-y_{i}=f_{i}(x) \in k(x)$. We wish to show that $y_{i}$ can be chosen so as to give $f_{i}(x)$ a particularly simple 
form. Any $\sigma \in G$ induces an automorphism of each field $k(x), K_{1}, \cdots, K_{n}$, and since $\sigma\left(P_{0}\right)=P_{0}$ we have $\sigma(x)=\alpha x+\beta$, with $\alpha, \beta \in k$. Since $\sigma^{p}=e, \alpha=1$, so $\sigma(x)=x+\beta$. $\sigma\left(y_{i}\right) \in K_{i}$ and $\sigma_{i}\left(\sigma\left(y_{i}\right)-y_{i}\right)=\sigma\left(\sigma_{i}\left(y_{i}\right)\right)-\sigma_{i}\left(y_{i}\right)=\sigma\left(y_{i}\right)-y_{i}$, so $\sigma\left(y_{i}\right)-y_{i}=g(x) \in k(x)$. But $\quad\left(\sigma\left(y_{i}\right)\right)^{p}-\sigma\left(y_{i}\right)=f_{i}(\sigma(x))$, so $\quad(g(x))^{p}-g(x)$ $=f_{i}(x+\beta)-f_{i}(x)$. There are an infinity of $\sigma$ 's so we can assume that $\sigma$ is chosen so that $P_{0}$ is the only pole that $f_{i}(x)$ and $f_{i}(x+\beta)$ can have in common. Use partial fractions to write $g(x)=g_{1}(x)+g_{2}(x)$, where $g_{1}(x)$ has poles only at the poles of $f_{i}(x)$ and $g_{2}(x)$ has no pole in common with $f_{i}(x)$. Then $\left(g_{1}(x)\right)^{p}-g_{1}(x)+f_{i}(x)=f_{i}(x+\beta)-\left(g_{2}(x)\right)^{p}+g_{2}(x)$ has poles only at $P_{0}$, that is $\left(g_{1}(x)\right)^{p}-g_{1}(x)+f_{i}(x) \in k[x]$. If we set $z_{i}=y_{i}+g_{1}(x)$ we get $\sigma_{i}\left(z_{i}\right)=z_{i}+1$ and $z_{i}^{p}-z_{i} \in k[x]$. Hence we may suppose $y_{i}$ chosen so that $f_{i}(x) \in k[x]$.

We digress for a moment to prove the following contention: If $u$ is an indeterminate and $\bar{k}(u, v)$ a field such that $v^{p}-v=f(u) \in \bar{k}[u]$, and if $\bar{k}(u, v) / \bar{k}$ has genus zero, then we can write $f(u)=(g(u))^{p}-g(u)+a u+b$, where $g(u)$ $\in \bar{k}[u]$ and $a, b \in \bar{k}$. First, if $v \in \bar{k}(u)$, then $v \in \bar{k}[u]$ and there is nothing to prove. So we may suppose that $[\bar{k}(u, v): \bar{k}(u)]=p$. Let $f(u)=c u^{r}+h(u)$, where $c \in \bar{k}, c \neq 0$, and where $h(u)$ has degree less than $r . r>0$. If $p \mid r$, say $r=p s$, then $\left(v-c^{1 / p} u^{s}\right)^{p}-\left(v-c^{1 / p} u^{s}\right)=h(u)+c^{1 / p} u^{s}$, and it clearly suffices to prove our contention with $f(u)$ replaced by $h(u)+c^{1 / p} u^{s}$, a polynomial of smaller degree. Repeating this process, we get that it suffices to prove the following: If $v^{p}-v=c u^{r}+h(u)$, where $c \in \bar{k}, c \neq 0, h(u) \in \bar{k}[u]$ of degree $<r$, and $r>1$ is prime to $p$, then $\bar{k}(u, v) / \bar{k}$ has genus $>0$. To do this consider the differential $d u$ of $\bar{k}(u, v)$. For any $\eta \in \bar{k}$ there are $p$ distinct places of $\bar{k}(u, v)$ lying over the place $(u=\eta)$ of $\bar{k}(u)$, so that $u-\eta$ is a uniformizing parameter at each of these places; hence $d u$ has order zero at each place of $\bar{k}(u, v)$ not lying over the place $(u=\infty)$ of $\bar{k}(u)$. But if $P$ is a place of $\bar{k}(u, v)$ such that $P(u)=\infty$, then $p v_{P}(v)=r v_{P}(u)$, so $v_{P}(u)=-p, v_{P}(v)=-r$. Hence $v_{P}(d u)=v_{P}\left(d v /\left(c r u^{r-1}+h^{\prime}(u)\right)\right.$ $=-r-1-(r-1)(-p)=(p-1)(r-1)-2$. This is $\geqq 0$ unless $p=2, r=2$, which case is excluded by the condition $p \nmid r$. Hence $d u$ is a nonzero differential of the first kind of $\bar{k}(u, v)$. This proves our contention.

Returning to our discussion of $K$, fix some $i(i=1, \cdots, n)$ and suppose that $f_{i}(x)$ has degree $N$. Write $f_{i}(x)=F\left(x^{p^{\nu}}\right)$ for some integer $\nu \geqq 0$, where $F$ is a polynomial. Setting $u=x^{p^{\nu}}, y_{i}^{p}-y_{i}=F(u) . \bar{k} K$ is rational, so by Lüroth's theorem so is $\bar{k}\left(u, y_{i}\right)$. By the above contention we can write $F(u)=(g(u))^{p}$ $-g(u)+a u+b$, where $g(u) \in \bar{k}[u]$ and $a, b \in \bar{k}$, and where we can assume $g(0)=0$. Hence $F^{\prime}(u)=-g^{\prime}(u)+a . F(u)$ has degree $N / p^{v}$ so (assuming $g(u) \neq 0) g(u)$ has degree $N / p^{v+1}$, and hence $F^{\prime}(u)$ has degree $\leqq N / p^{v+1}-1$. Hence we can find a polynomial $H(u) \in k[u]$ of degree $\leqq N / p^{\nu+1}$ such that $H^{\prime}(u)=F^{\prime}(u)$. Writing $z_{i}=y_{i}+H(u)$ we get $\sigma_{i}\left(z_{i}\right)=z_{i}+1$ and $z_{i}^{p}-z_{i}=F(u)$ $+(H(u))^{p}-H(u)=$ a polynomial in $k[u]$ of degree $\leqq N / p^{\nu}$ with derivative zero. Hence $z_{i}^{p}-z_{i}=G\left(x^{p^{p+1}}\right)$, where $G\left(x^{p^{p+1}}\right)$ is a polynomial of degree $\leqq N$ with coefficients in $k$. Hence we could have assumed to begin with that $f_{i}(x)$ 
is a polynomial in $x^{p^{p+1}}$, and repeat this process, if possible, to replace $f_{i}(x)$ by another polynomial of degree $\leqq N$ that is a polynomial in $x^{p^{p+2}}$, etc. This process must come to an end, so finally we get $g(u)=0$. Then $y_{i}^{p}-y_{i}=a x^{p^{\nu}}+b$, with $a, b \in k$. If $\nu>0$ and $a \in k^{p}$, then $\left(y_{i}-a^{1 / p} x^{p^{p-1}}\right)^{p}-\left(y_{i}-a^{1 / p} x^{p^{\nu-1}}\right)$ $=a^{1 / p} x^{p^{\nu-1}}+b$, so if we choose $\nu$ minimal we have either $\nu=0$ or $a \notin k^{p}$. But if $\nu=0$, then $y_{i}^{p}-y_{i}=a x+b$, so $K_{i}$ is a rational field with the place under $P$ rational, contradicting the minimality of $n$. Hence we can assume that for $i=1, \cdots, n$ we have $y_{i}^{p}-y_{i}=a_{i} x^{p^{m}}{ }_{i}+b_{i}$, with $a_{i}, b_{i} \in k, a_{i} \notin k^{p}$, and $m_{i}>0$. The only automorphism of $G_{0}$ leaving each $y_{i}$ fixed is $e$, so $K=k\left(x, y_{1}, \cdots, y_{n}\right)$. For any $\sigma \in G$ we have $\sigma(x)=x+\beta, \beta \in k$. Setting $\alpha_{i}=\sigma\left(y_{i}\right)-y_{i}$, we get $\alpha_{i}^{p}-\alpha_{i}=a_{i} \beta^{p^{m}}$, so $\alpha_{i} \in k$. Conversely suppose $\alpha_{1}, \cdots, \alpha_{n}, \beta \in k$ and that $\alpha_{i}^{p}-\alpha_{i}=a_{i} \beta^{p^{m}}, i=1, \cdots, n$. Let $X, Y_{1}, \cdots, Y_{n}$ be indeterminates. Then the prime ideal in $k[X, Y]$ having $\left(x, y_{1}, \cdots, y_{n}\right)$ as generic zero is generated by the various polynomials $\left(Y_{i}^{p}-Y_{i}-a_{i} X^{p^{m_{i}}}-b_{i}\right)$, so setting $\sigma(X)=X+\beta$, $\sigma\left(Y_{i}\right)=Y_{i}+\alpha_{i}, i=1, \cdots, n$, gives an automorphism of this ring carrying our prime ideal onto itself, and hence we get an automorphism $\sigma$ of $k\left[x, y_{1}, \cdots, y_{n}\right]$ (and hence of $K$ ) such that $\sigma(x)=x+\beta, \sigma\left(y_{i}\right)=y_{i}+\alpha_{i}$. We summarize as follows.

Theorem. Let $K / k$ satisfy the exceptional case. Then there exist $x \in K$ such that $[K: k(x)]=p^{n}$, where $p \neq 0$ is the characteristic of $K$, elements $y_{1}, \cdots, y_{n}$ $\in K, a_{1}, \cdots, a_{n}, b_{1}, \cdots, b_{n} \in k$, with $a_{1}, \cdots, a_{n} \notin k^{p}$, and strictly positive integers $m_{1}, \cdots, m_{n}$ such that $K=k\left(x, y_{1}, \cdots, y_{n}\right)$ and $y_{i}^{p}-y_{i}=a_{i} x^{p^{m}}{ }_{i}+b_{i}$, $i=1, \cdots, n$. For each set of elements $\beta, \alpha_{1}, \cdots, \alpha_{n} \in k$ such that $\alpha_{i}^{p}-\alpha_{i}$ $=a_{i} \beta^{p^{m}} ;, i=1, \cdots, n$, we have an automorphism $\sigma$ of $K / k$ defined by $\sigma(x)=x$ $+\beta, \sigma\left(y_{i}\right)=y_{i}+\alpha_{i}, i=1, \cdots, n$, and the set of all such automorphisms $\sigma$ forms the normal subgroup $G$ of the full group of automorphisms $G$ of $K / k$ such that $G / G$ is cyclic of finite order prime to $p$.

It is easy to establish a converse of this theorem: Let $a_{1}, \cdots, a_{n}$, $b_{1}, \cdots, b_{n}, m_{1}, \cdots, m_{n}$ be as above and let $K$ be the splitting field over $k(x)$ of the polynomial $\prod_{i=1}^{n}\left(Y^{p}-Y-a_{i} x^{p^{m}}{ }_{i}-b_{i}\right)$. By deleting some of the factors in the product if necessary, we can assume that $[K: k(x)]=p^{n}$. If we have an infinite number of sets $\beta, \alpha_{1}, \cdots, \alpha_{n} \in k$ satisfying $\alpha_{i}^{p}-\alpha_{i}=a_{i} \beta^{p^{m}}{ }_{i}, i=1, \cdots$, $n$, and if $K$ has genus $>0$, then $K / k$ satisfies the exceptional case.

If $K / k$ is exceptional, then its genus $g$ cannot be arbitrary. First, since the genus drops to zero when we extend $k$ to $\bar{k}$, by a result of Tate [6] g must be a multiple of $(p-1) / 2$. Second, Zeuthen's formula $2 g-2=-2 p^{n}+r d(P)$ implies $2 g-2 \equiv 0(p)$. Thus $g$ is of the form $g=(s p-2)(p-1) / 2$, where $s$ is an integer. For example, if $K=k(x, y)$, where $y^{p}-y=a x^{p}, a \in k, a \notin k^{p}$, then the curve in the projective plane whose generic point over $k$ is $(1, x, y)$ is immediately seen to be nonsingular with reference to $k$, so in this case $g$ $=(p-1)(p-2) / 2$. This last field $K$ is clearly exceptional if $p>2$ and $k$ is separably algebraically closed. 


\section{REFERENCES}

1. P. Appell and E. Goursat, Theorie des fonctions algébriques et de leurs integrales, Paris, 1895.

2. C. Chevalley, Introduction to the theory of algebraic functions of one variable, Mathematical Surveys, no. 6, 1951.

3. K. Iwasawa and T. Tamagawa, On the group of automorphisms of a function field, J. Math. Soc. Japan vol. 3 (1951) and vol. 4 (1952).

4. M. Rosenlicht, Equivalence relations on algebraic curves, Ann. of Math. vol. 56 (1952).

5. H. L. Schmid, Über die Automorphismen eines algebraischen Funktionenkörpers von Primzahlcharacleristik, J. Reine Angew. Math. vol. 179 (1938).

6. J. Tate, Genus change in inseparable extensions of function fields, Proc. Amer. Math. Soc. vol. 3 (1952).

7. A. Weil, Varietes abeliennes et courbes algebriques, Paris, 1948.

8. O. Zariski, The concept of a simple point of an abstract algebraic variety, Trans. Amer. Math. Soc. vol. 62 (1947).

\section{NORTHWESTERN UNIVERSITY,} Evanston, Ill. 